\title{
Diversity of Urinary Tract Pathogens and Drug Resistant Isolates of Escherichia Coli in different age and gender Groups of Pakistanis
}

\author{
MF Bashir ${ }^{1 *}$, JI Qazi ${ }^{2}$, N Ahmad ${ }^{2}$, and S Riaz ${ }^{3}$ \\ ${ }^{1}$ The School of Biological Sciences, ${ }^{2}$ Department of Zoology, ${ }^{3}$ Microbiology and Molecular Genetics, University of the \\ Punjab, Lahore 54590, Pakistan.
}

\begin{abstract}
Purpose: This paper was mainly aimed to investigate drug resistance of the various urinary tract infection (UTI) pathogens from patients of different gender and age groups of Pakistanis.

Method: For these purposes, urine samples of 109 patients were analyzed. Samples were screened on CLED agar. Antimicrobial susceptibility testing was performed by Kirby Bauer's disc diffusion method. Isolated colonies were processed for biochemical characterization and antibiotic sensitivity to ampicillin, amikacin, augmentin, ceftazidime, ceftriaxone, cefuroxime, cotrimoxazole, ciprofloxin, imipenem, meropenem, tazocine, trimethoprim, gentamicin and nitrofuratoin.

Result: E.Coli was found to be the most frequent causative agent of UTIs (66\%) followed by Enterococci (8.3\%), Candida spp. and Pseodomonas spp. (7.3\% each), Klebsiella spp. (5.5\%) and Enterobacter spp. (2.7\%). Proteus. and Morgenella species were found in less than $1 \%$ of the cases. E.coli showed variable antimicrobial resistance to different antibiotics as $92 \%, 86 \%, 80 \%, 62 \%, 47 \%$, $20 \%$ and $4 \%$ of the isolates were found to be resistant to ampicillin, cotrimoxazole, ciprofloxin, gentamicin, nitrofuratoin and amikacin, respectively.

Conclusion: The most effective in vitro agents were found to be amikacin followed by gentamicin (among the parenterals), and ciprofloxin among the orally administratered ones. A higher prevalence of UTIs was observed in the female population and E.coli showed no resistance to nitrofuratoin in age groups of $50^{+}$and $70^{+}$in both genders.
\end{abstract}

Keywords: Urinary tract infections, Age, Gender, Resistant microbes, E.coli. 


\section{INTRODUCTION}

With the constantly shifting trends in drug resistance, antibiotic options, and pharmacoeconomic considerations, urinary tract infections (UTIs) continue to be one of the most frequently diagnosed cases, having an estimated figure of 150 million per annum worldwide. In fact, UTIs are the leading cause of gram-negative bacteremia in patients of all ages and are associated with a high risk of morbidity and mortality, especially in the elderly, and account for significant health care costs. ${ }^{1,2}$ Warren et al $^{3}$ have reported that in the United States, urinary tract infections result in approximately 8 million physician visits per year.

In spite of the availability and use of the antimicrobial drugs, UTIs caused by bacteria have been showing increasing trends in recent years. Much of the increase has been related to emerging antibiotic resistance in urinary tract pathogens. ${ }^{4}$ The common uropathogens identified in adult patients with UTIs include enteric gram-negative bacteria, with $E$. coli being the most common. The remainders of infections are caused by coagulase-negative

Staphylococcus

saprophyticus (10-20\%), while Proteus mirabilis, Klebsiella, and Enterococcus account for less than $5 \%$. Other aerobic gram-negative bacteria of the Enterobacteriacea family include Citrobacter, Enterobacter, Serratia, and Salmonella, .

The high incidence of UTIs in the general population, the potential for complications, especially in high-risk subgroups and the associated costs of treatments emphasize the importance of appropriate antibiotic therapy. Microbial resistance to nearly all classes of antimicrobials continues to rise despite increasing awareness and concerns worldwide ${ }^{4,8,9,10-12}$

The situation is more adverse where indiscriminate use of antibiotics is rampant. In Pakistan, general practitioners, at large, recommend antibiotics without isolation and sensitivity test for pathogens. Poverty and illiteracy further strengthen the indiscriminate use of antibiotics including the low/high dose intake, incomplete course and a general trend of using left over drugs. Such practices may result an increase in resistant pathogens. The present report describes some data in this regard. For this purpose one hundred and nine positive urine samples received at Shaukat Khanum cancer hospital, Lahore were employed to isolate and characterize the pathogens for UTIs and to make comparison of antibiotics sensitivity/ resistance patterns. These data provide baseline information for further study and are useful for managing UTI cases.

\section{METHODS Study Design}

This study focuses on the antibiotic susceptibility patterns and frequency of uropathogens in different gender and age groups. 109 positive human urine samples obtained from different collection points and hospitalized patients at Shaukat Khanum cancer hospital and research center, Lahore were used for this purpose. These samples were processed to monitor urinary tract infection and antibiotics sensitivity patterns of pathogenic bacteria. The data were categorized viz gender and age. This study was approved from the University of the Punjab. Lahore.

\section{Study Protocols}

The specimens were collected for about three months between February 2004 to April 2004. The specimens which grew positive were selected for further analysis.

The specimens which grew more then one type of colonies were excluded from this study. The specimens whose age or sex record were not available were also rejected. A bacterial colony count of $10,000 \mathrm{cfu} / \mathrm{ml}$ and $100,000 \mathrm{cfu} / \mathrm{ml}$ of urine for patients $0-16$ years and above 16 years respectively, was selected as criterion for inclusive in the study. It is identical to that used to diagnose UTI in a number of previous studies ${ }^{4,8}$. Lactobacilli and coagulase-negative Staphylococci were considered as contaminants and were also excluded from the study. 
The urine samples were inoculated on cysteine lactose electrolyte deficient agar (CLED). All Gram negative rods and enterococci were identified by using conventional biochemicals plus API 20E (Biomeurex) and Oxoid Streptococcal grouping kits, respectively. Antibiotic sensitivity testing of all isolates was performed on muller hinton agar plates by Kerby-Baure disk diffusion technique with guidelines established by National Committee for Clinical Laboratory Standards ( NCCLS $)^{13}$ Oral antibiotics for which uropathogens were tested include; ampicillin, trimethoprime, ciprofloxin, nitrofuratoin, augmentin, and cotrimoxazole.Parental antibiotics include; gentamicin, amikacin, imipenem, meropenem, , ceftazidime, ceftriaxone, cefuroxime, and Tazocin.

\section{Data Analysis}

Data were categorized viz age and sex. The patients were divided into age groups as $0_{+}$ (0-9 years), $10+(10-19$ years), $20+(20-29$ years), $30+(30-39$ years), $40+$ (40-49 years), $50+$ (50-59 years), $60+(60-69$ years $), 70+$ (70-79 years), $80+(80-89$ years) and $90+(90-$ 99 years).

\section{RESULTS}

When the data were classified into different age and gender groups, it appeared that the cases of urinary tract infections (UTIs) were more in boys than girls of less than ten years of age. For the age group $20+$ the females showed more than double the \% of UTIs cases in males. For the next age group $(30+)$ the situation appeared inverse to the preceding age group. Then for the age groups $40+$ and $60+$ females had $6.64 \%$ and $6.73 \%$ higher prevalence of the infection respectively than the corresponding male groups. However for age group 50+ gender wise difference was not prominent. For $70+$ age group the cases for males appeared double than the females (Table 1).

Among the uropathogens E.coli was the most frequent in both sexes with $44.45 \%$ and $78.1 \%$ frequencies in male and female patients, respectively. Enterococci caused two folds $(9.6 \%)$ UTIs in females than the males (5.55\%). Candida, Pseudomonas and Klebsiella were isolated from $13.89 \%, 13.89$ $\%$ and $11.11 \%$ of the males, respectively. For females the corresponding figures were 4.1 $\%, 4.1 \%$ and $2.74 \%$, respectively. The uropathogens, nterobacter $(8.33 \%)$ and Morganella (2.78 \%) appeared only in males. UTI cases caused by Proteus appeared 1.36 $\%$ in females only (Table 2 ).

When sensitivity to different antibiotics was compared, imipenen, meropenum and tazocin were found effective against the bacterial isolates from both sexes. It is surprising that all the antibiotics, except amikacin, showed more resistance in male patients than females. Nitrofuratoin showed about two folds resistance in males than females, while Trimethoprim, cotrimoxazole and ceftazidime showed $11 \%$ more resistance in males than females. Ceftriaxone, ciprofloxin, ampicillin and augmentin showed 13\%,14\%, 16\% and $20 \%$ more resistance in males as compared to females, respectively (Table 3).

E.coli did not show resistance to imipenen, meropenum and tazocin in all age groups of males. On the other hand the bacteria manifested almost complete resistance to ampicillin, trimethoprim and cotrimoxazole in all the age groups. The isolates from below 40 years male patients and age groups $50+$ and 70+ showed almost complete resistance to ciprofloxin, while it was effective in half of male patients in age groups $40+$ and $60+$. Amikacin experienced little resistance in age groups $20+(16.66 \%), 40+(8.33 \%), 50+(10 \%)$ and $70+(16.66 \%)$, while for other age groups it was completely effective. Different trends in resistance for the different age groups are shown in this study.

Bacterial isolates from females showed relatively less resistance than males. Imipenem, tazocin, meropenum and amikacin were found effective in most of the age groups, while these drugs showed $16.66 \%$ resistance in age group 20+. Nitrofurantoin showed $33 \%$ resistance in age groups $0+, 20+$ and $30+$ and was found almost sensitive in all other age' groups. Ampicillin faced almost 
Bashir et al

Table 1: Prevalence of UTIs in males and females of different age groups

\begin{tabular}{ccccc}
\hline Age groups (Years) & Male & $(\%)$ & Female & $(\%)$ \\
\hline $0+$ & $5^{\mathrm{a}}$ & 13.88 & 9 & 12.33 \\
$10_{+}$ & 2 & 5.55 & 5 & 6.85 \\
$20+$ & 2 & 5.55 & 10 & 13.69 \\
$30+$ & 5 & 13.88 & 4 & 5.48 \\
$40+$ & 4 & 11.17 & 13 & 17.81 \\
$50_{+}$ & 7 & 19.44 & 14 & 19.19 \\
$60_{+}$ & 3 & 8.33 & 11 & 15.06 \\
$70+$ & 5 & 13.88 & 6 & 8.22 \\
$80+$ & 1 & 2.77 & 0 & 0 \\
$90+$ & 2 & 5.55 & 1 & 1.37 \\
\hline Total & 36 & 100 & 73 & 100 \\
\hline \multicolumn{2}{c}{ Number of cases representing a given age group out of total 109 cases. }
\end{tabular}

Table 2: Prevalence of different UTI pathogens among male and female patients

\begin{tabular}{lccc}
\hline Pathogens & Male & Female & Total no. of isolates \\
\hline Candida & $5(13.89)^{\mathrm{a}}$ & $3(4.1)$ & $8(7.35)$ \\
E.coli & $16(44.45)$ & $57(78.1)$ & $73(66.97)$ \\
Enterobacter & $3(8.33)$ & $0(0)$ & $3(2.75)$ \\
Enterococci & $2(5.55)$ & $7(9.6)$ & $9(8.26)$ \\
Klebsiella & $4(11.11)$ & $2(2.74)$ & $6(5.5)$ \\
Morganella & $1(2.78)$ & $0(0)$ & $1(0.91)$ \\
Proteus & $0(0)$ & $1(1.36)$ & $1(0.91)$ \\
Pseudomonas & $5(13.89)$ & $3(4.1)$ & $8(7.35)$ \\
\hline Total & $36(100)$ & $73(100)$ & $109(100)$ \\
\hline
\end{tabular}

${ }^{a}$ Values in parenthesis indicate the percentage of the isolate for a given gender.

complete resistance in all age groups except $0+, 20,+$ and $70+$ for which $67 \%$ resistance was recorded. Augmentin showed variation in resistance from $25 \%$ to $70 \%$ in different age groups and was found to be sensitive in age groups $10+$ and 20+. Ceftriaxone showed
$60 \%$ resistance in age group $60+$. ceftriaxone was sensitive in age group 10+, while it showed variable resistance among other age groups. Ciproflaxin, cotrimoxazole and trimethoprim showed variable resistance patterns in all age groups except $40+$ in which 
these antibiotics were effective among half the female patients. (see Table 4). In general, E.coli isolates in this study showed varying patterns of antimicrobial resistance to different antibiotics viz ampicillin about (92\%), trimethoprim (86\%) cotrimoxazole $(80 \%)$, ciprofloxin $(62 \%)$, gentamicin, nitrofuratoin (20\%) and amikacin (4\%).

Enterococci, the only gram positive isolate, was found to be susceptible to ampicillin, imipenem, penicillin, tazocin and vancomycin. The Enterococci isolates showed resistance to ceftriaxone $(77.77 \%)$ and cefuroxime (66.66\%).

\section{DISCUSSION}

The most frequent causative agent of UTIs in this study was found to be E.Coli $(66.97 \%)$ followed by Enterococci (8.26\%), Candida spp. and Pseodomonas spp. $(7.34 \%$ each),Klebsiella spp.(5.50\%), Enterobacter spp. $(2.75 \%)$, Proteus spp. and Morgenella spp.(0.91\% each). In two other studies in Pakistan, Khan $^{6}$ reported a uropathogens prevalence of $45.6 \%$ for E. coli. This was followed by Candida spp. (10.5\%), Enterococcus spp. (10.2\%) while Farooqy ${ }^{14}$ revealed a prevalence of $42 \%$ for $E$. coli, $16 \%$ Pseudomonas aeruginosa, 11\% Klebsiella aerogenes, 5.0\% ,Enterobacter spp., 13\% Proteus spp., $4.0 \%$ Serratia liquifaciens, $1.0 \%$,Acinetobacter spp., $3.0 \%$ Citrobacter spp., $\quad 4.0 \% \quad$ Enterococci and $0.5 \%$ Staphylococcus aureus. Prevalence of different pathogens is dependent on several population attributes, sample size and hygienic conditions of the patients. Therefore, a stable pattern in this regard cannot be predicted. This is why data vary when different studies are compared. For example, Hsueh et $\mathrm{al}^{15}$ have reported that the most frequent isolates from UTIs at a university hospital in Taiwan during the period 1993 - 1998 were Candida spp. (23.6\%), E.Col i(18.6\%) and P.aeruginosa (11.0\%). However, in 1999, E.Coli (18.4\%) replaced Candida spp. (14.3\%) as the top ranking pathogen causing UTIs.

The consistently high prevalence of resistance to cotrimoxazole and an increasing pattern of resistance to ciprofloxacin require ongoing surveillance to identify further changes among urinary tract isolates of $E$. coli. ${ }^{16}$ The in vitro activities of nitrofuratoin and the other antibiotics with even lesser resistance found in the present study suggest that they would provide adequate alternative therapy in locations where the uropathogens E.coli had developed multi drugs resistance ${ }^{17}$ Planning to reduce resistance is very important, as organisms resistant to one antimicrobial agent are likely to become resistant to others. Researchers have found that bacteria isolated from UTIs have resistance to common drugs such as ampicillin and sulphonamides in over one-third of cases. These drugs have been on the market for several decades ${ }^{16}$

In the present study when the data were visualized in different age groups for both genders, it appeared that in the case of males, E.coli isolates were susceptible to nitrofuratoin in age groups $0_{+}, 50+$ and $70_{+}$, while in age groups $20+, 30+$ and $60+$ hundred percent resistance was recorded. E.Coli In females, showed no resistance to nitrofuratoin in age groups $10_{+}, 50+$ and $70+$, while it showed $33 \%$ resistance in age groups $0+, 20+$ and $30+$. E. Coli isolates from age groups $50+$ and $70+$ both in males and females were found to be $100 \%$ susceptible to the nitrofuration.(Table 4) Such varying pattern of resistance also appeared in different age and gender groups to other antibiotics in this study. These findings are difficult to explain. McLoughlin and Joseph ${ }^{18}$ have indicated that prevalence of resistant organisms vary by geography, patient age, and hospitalization status. The present analysis indicates the more likelihood of selective development of resistance to some antibiotics in certain age groups may have link(s) with physiological differences amongst the groups. However, such data appear beneficial to physician for empirical prescriptions of different antibiotics taking into account many age groups. The activity of the antibiotics investigated in this study against E.coli in female patients are as follows, in decreasing order: imipenem = meropenum $>$ tazocin $>$ amikacin $>$ nitrofurantoin $>$ ceftazidime $>$ ceftriaxone $>$ 
Table3: Prevalence (\%) of antibiotics resistance in E.coli isolated from males and females

\begin{tabular}{lccc}
\hline Antibiotics & Males & Females & Total \\
\hline Ampicillin & & & 92 \\
Augmentin & 100 & 84.21 & 58 \\
Ceftazidime & 68.75 & 47.36 & 35 \\
Ceftriaxone & 43.75 & 26.31 & 50 \\
Cefuroxime & 56.25 & 43.85 & 60 \\
Imipenem & 68.75 & 49.12 & 1.75 \\
Meropenum & 0 & 1.75 & 1.75 \\
Tazocin & 0 & 1.75 & 3.5 \\
Amikacin & 0 & 3.5 & 7 \\
Gentamicin & 0 & 7.01 & 49 \\
Ciproflaxin & 50 & 47.36 & 62 \\
Cotrimoxazole & 68.75 & 54.38 & 80 \\
Trimethoprim & 81.25 & 78.94 & 86 \\
Nitrofuratoin & 93.75 & 78.94 & 20 \\
\hline
\end{tabular}

Table 4: Prevalence (\%) of E.coli resistance to different antibiotics isolated from males of and females of different age groups

\begin{tabular}{ccccccccc}
\hline & \multicolumn{4}{c}{ Antibiotics } & \multicolumn{4}{c}{ Age in years } \\
\cline { 2 - 9 } Ampicillin & $0+$ & $10+$ & $20+$ & $30+$ & $40+$ & $50+$ & $60+$ & $70+$ \\
& 100 & NSR & 100 & 100 & 100 & 100 & 100 & 100 \\
Augmentin & $(66.66)$ & $(100)$ & $(66.66)$ & $(100)$ & $(83.33)$ & $(90)$ & $(100)$ & $(66.66)$ \\
& 100 & NSR & 100 & 100 & 100 & 60 & 60 & 40 \\
Ceftazidime & $(50)$ & $(25)$ & $(33.33)$ & $(66.66)$ & $(33.33)$ & $(70)$ & $(60)$ & $(66.66)$ \\
& 100 & NSR & 0 & 0 & 100 & 40 & 100 & 20 \\
Ceftiaxone & $(33.33)$ & $(0)$ & $(0)$ & $(33.33)$ & $(33.33)$ & $(30)$ & $(30)$ & $(33.33)$ \\
& 100 & NSR & 100 & 0 & 100 & 40 & 100 & 20 \\
Cefuroxime & $(50)$ & $(0)$ & $(16.66)$ & $(33.33)$ & $(41.66)$ & $(50)$ & $(60)$ & $(33.33)$ \\
& 100 & NSR & 100 & 100 & 100 & 60 & 100 & 40 \\
Imipenem & $(66.66)$ & $(25)$ & $(16.66)$ & $(66.66)$ & $(41.66)$ & $(50)$ & $(70)$ & $(50)$ \\
& 0 & NSR & 0 & 0 & 0 & 0 & 0 & 0 \\
Meropenum & $(0)$ & $(0)$ & $(16.66)$ & $(0)$ & $(0)$ & $(0)$ & $(0)$ & $(66.66)$ \\
& 0 & NSR & 0 & 0 & 0 & 0 & 0 & 0 \\
Tazocin & $(0)$ & $(0)$ & $(16.66)$ & $(0)$ & $(0)$ & $(0)$ & $(0)$ & $(0)$ \\
& 0 & NSR & 0 & 0 & 0 & 0 & 0 & 0 \\
Nitrofuratoin & $(0)$ & $(0)$ & $(16.66)$ & $(33)$ & $(0)$ & $(0)$ & $(0)$ & $(16.66)$ \\
& 0 & NSR & 100 & 100 & 50 & 0 & 100 & 0 \\
Amikacin & $(33.33)$ & $(0)$ & $(33.33)$ & $(33)$ & $(8.33)$ & $(0)$ & $(10)$ & $(0)$ \\
& 0 & 0 & 16.66 & 0 & 8.33 & 10 & 0 & 16.66 \\
Gentamicin & $(0)$ & $(0)$ & $(16.66)$ & $(0)$ & $(8.33)$ & $(10)$ & $(0)$ & $(16.66)$ \\
& 66.66 & 25 & 33.33 & 33 & 41.66 & 30 & 70 & 83.66 \\
& $(66.66)$ & $(25)$ & $(33.33)$ & $(33)$ & $(41.66)$ & $(30)$ & $(70)$ & $(83.66)$ \\
Ciproflaxin & 100 & NSR & 100 & 100 & 50 & 80 & 40 & 100 \\
Cotrimoxazole & $(50)$ & $(50)$ & $(16.66)$ & $(66.66)$ & $(50)$ & $(60)$ & $(70)$ & $(50)$ \\
& 100 & NSR & 100 & 100 & 100 & 100 & 100 & 60 \\
Trimethoprim & $(83.33)$ & $(100)$ & $(83.66)$ & $(100)$ & $(50)$ & $(90)$ & $(80)$ & $(66.66)$ \\
& 100 & NSR & 100 & 100 & 100 & 100 & 100 & 80 \\
& $(83.3)$ & $(100)$ & $(100)$ & $(100)$ & $(50)$ & $(80)$ & $(80)$ & $(83.66)$ \\
\hline Val & & $(100)$ & & &
\end{tabular}

Values within parentheses indicate female patients; $N S R=$ no sample received

gentamicin $=$ augmentin $>$ cefuroxime $>$ ampicillin. For isolates from male patients, as ciprofloxin $>$ cotrimoxazole $=$ trimethoprim $>$ the order is: imipenem $=$ meropenum $=$ 
tazocin $=$ amikacin $>$ nitrofuratoin $>$ ceftazidime > gentamicin > ceftriaxone > augmentin $=$ Cefuroxime $=$ ciprofloxin $>$ cotrimoxazole $>$ trimethoprim $>$ ampicillin.

Similar orders can be worked out for isolates with respect to different age groups. These resistance and susceptibility patterns should be considered in order to improve empirical antibiotic therapy in UTI cases.

\section{CONCLUSION}

The organisms showed resistance to older urinary antimicrobial agents such as ampicillin and cotrimoxazole; this indicates that increased consumption of a particular antibiotic can be a pathway to its resistance. Higher prevalence of UTIs was observed in female population. The prevalence of UTIs was highest for age group $50+$ followed by $0+$ in both genders. E.coli showed no resistance to nitrofuratoin in age groups $50+$ and $70+$ for both genders.

\section{ACKNOWLEDGMENT}

The authors wish to thank the staff of the Microbiology and Molecular Biology section, Department of Pathology, Shaukat Khanum Memorial Cancer Hospital, Lahore, Pakistan, for their assistance in the experimental work.

\section{REFERENCES}

1. Orenstein $R$, Wong ES. Urinary tract infections in adults. Am Fam Physician.1999; 59:1225-1234.

2. Stamm WE, Norrby SR. Urinary tract infections: disease panorama and challenges. J Infect Dis. 2001; 183(Supp/ 1): S1-S4.

3. Warren JW, Abrutyn E, Hebel JR, Johnson JR, Schaeffer AJ, Stamm WE. Guidelines for antimicrobial treatment of uncomplicated acute bacterial cystitis and acute pyelonephritis in women. Clin Infect Dis. 1999; 29: 745-758.

4. Newell A, Riley $P$, Rogers $M$. Resistance patterns of urinary tract infections diagnosed in a genitourinary medicine clinic. Int J STD AIDS .2000;11:499-500.

5. Baerheiy A, Digranes A, Hunskar S. Are resistance patterns published by microbiological laboratories valid for general practice? APMIS. 1999; 107:676-680.
6. Khan SW, Ahmed A. Uropathogens and their susceptibility pattern: a retrospective analysis.J Pak Med Assoc. 2001; 51:98-100

7. Steele RW. The epidemiology and clinical presentation of urinary tract infections in children 2 years of age through adolescence. Pediatr. Ann.1999; 28:653-658.

8. Akram $M$, Shahid $M$, Khan AU. Etiology and antibiotic resistance patterns of communitycquired urinary tract infections in $J N M C$ Hospital Aligarh, India. Ann Clin Microb Antimicrob. 2007; 6:4.

9. Beckford-Ball J: Related Articles, Management of suspected bacterial urinary tract infection. Nurs Times. 2006; 102:25-6.

10. Belongia EA, Schwartz B. Strategies for promoting judicious use of antibiotics by doctors and patients. BMJ.1998; 317:668-671.

11. Landhani $S$, GrandsdenW. Increasing antibiotics resistance among urinary tract isolates. Arch Dis child.2003; 88:444-5.

12. Talan DA, Stamm WE, Hooton TM, Moran GJ, Burke $T$, Iravani $A$, Reuning-Scherer J, Church DA. Comparison of ciprofloxacin (7 days) and trimethoprim-sulfamethoxazole (14 days) for acute uncomplicated pyelonephritis in womena randomized trial. JAMA 2000; 283:15831590.

13. National Committee for Clinical Laboratory Standards. Performance standards for antimicrobial susceptibility testing. International Supplement. NCCLS Committee for Clinical Laboratory Standards. Wayne, Pa 11th edition. 2001.

14. Farooqui $B J$, Khurshid $M$, Alam M. Urinary tract infection, J Pak Med Assoc. 1989; 39:129-31.

15. Hsueh $P$, Chen $M$, Sun $C$, Chen $W$, Pan $H$, Yang $L$, Chang S. Antimicrobial drug resistance in pathogens causing nosocomial infections at a university hospital in Taiwan: Emerg Infec Dis.2002; 8:63-68.

16. Karlowsky JA, Kelly LJ, Thornsberry C, Jones ME, Sahm, D.F. Trends in Antimicrobial Resistance among Urinary Tract Infection Isolates of Escherichia coli from Female Outpatients in the United States; Antimicrob Agents Chemother.2002; 46: 2540-2545

17. Olafsson, M, Kristinsson KG, Sigurdsson JA.Urinary tract infections, antibiotic resistance and sales of antimicrobial drugs; An observational study of uncomplicated urinary tractinfections in Icelandic women.Scand $J$ Prim Health Care.2000;18:35-38.

18. McLoughlin, TG, Jr, Joseph MM. Antibiotic Resistance Patterns of Uropathogens in Pediatric Emergency Department Patients. Acad Emerg Med. 2003;10:347-351 